\title{
Photon-counting linear discriminant analysis for face recognition at a distance
}

\author{
Seokwon Yeom* \\ Division of Computer and Communication Engineering, Daegu University, Gyeongsan, Gyeongbuk, $712-714$ \\ South Korea
}

\begin{abstract}
Face recognition has wide applications in security and surveillance systems as well as in robot vision and machine interfaces. Conventional challenges in face recognition include pose, illumination, and expression, and face recognition at a distance involves additional challenges because long-distance images are often degraded due to poor focusing and motion blurring. This study investigates the effectiveness of applying photon-counting linear discriminant analysis (Pc-LDA) to face recognition in harsh environments. A related technique, Fisher linear discriminant analysis, has been found to be optimal, but it often suffers from the singularity problem because the number of available training images is generally much smaller than the number of pixels. Pc-LDA, on the other hand, realizes the Fisher criterion in high-dimensional space without any dimensionality reduction. Therefore, it provides more invariant solutions to image recognition under distortion and degradation. Two decision rules are employed: one is based on Euclidean distance; the other, on normalized correlation. In the experiments, the asymptotic equivalence of the photon-counting method to the Fisher method is verified with simulated data. Degraded facial images are employed to demonstrate the robustness of the photon-counting classifier in harsh environments. Four types of blurring point spread functions are applied to the test images in order to simulate long-distance acquisition. The results are compared with those of conventional Eigen face and Fisher face methods. The results indicate that Pc-LDA is better than conventional facial recognition techniques.
\end{abstract}

Keywords : Pattern recognition, Face classification, Statistical classification, Photon-counting, Linear discriminant analysis.

\section{Introduction}

Face classification has many applications in security monitoring, intelligent surveillance, robot vision, humanmachine interface, and image retrieval [1, 2]. It is challenging to classify a facial image acquired in an uncontrolled setting like long distance. Various classifiers have been developed on the basis of statistical analyses [1-9]. Among them, Fisher Linear discriminant analysis (LDA) is a popular technique because it enables the maximization of the discrimination capability through the Fisher criterion [10]. However, Fisher LDA often encounters the singularity problem of within-class covariance matrix, which is often called the "small sample size (SSS) problem" [11]. The SSS problem occurs in most practical cases, when the number of pixels is larger than the number of available training images. Several approaches have been applied to overcome this problem, including dimensionality reduction using principal component analysis (PCA) [3, 4], regularized LDA methods [5, 6], and replacement of the inverse of the within-class matrix with a pseudo-inverse matrix [7]. However, these approaches cannot ensure the optimality of the Fisher criterion.

Manuscript received Dec. 16, 2011; revised Jun. 5, 2012; accepted Aug. 12, 2012

* Corresponding author: yeom@daegu.ac.kr

This research was supported by Basic Science Research Program through the National Research Foundation of Korea (NRF) funded by the Ministry of Education, Science and Technology (No. 2011-0003853 and 2012R1A1A2008545).

(C) The Korean Institute of Intelligent Systems. All rights reserved.
In this paper, photon-counting (Pc) LDA is applied to facial image recognition in harsh environments. Pc-LDA does not suffer from the singularity problem because it realizes the Fisher criterion with the Poisson probability model [12, 13]. Pc-LDA was proposed for the classification of photon-limited images obtained under low illumination.

In the original Pc-LDA, intensity images are trained to build the classifier, and the class decision is made by a photonlimited image. In this study, Euclidean distance- and normalized correlation-based decision rules are used to classify the intensity image, which is derived by a Bayesian estimation of the photon-limited image. One advantage of Pc-LDA is that, unlike Fisher LDA, it does not involve dimensionality reduction [3, 4]. Another advantage is that the classifier is robust to distortion, such as blurring and noise corruption, because the Fisher criterion is realized in high-dimensional space.

In the experiments, simulated data are used to verify the asymptotic equivalence of Pc-LDA to Fisher LDA. Test images are degraded by applying defocusing and motion blurring point spread functions (PSFs) in order to simulate image acquisition at a distance. The discrimination capability is evaluated by the average detection $\left(P_{D}\right)$ and false alarm $\left(P_{F A}\right)$ probabilities. The performance is compared with that of conventional methods, such as Eigen face (PCA) and Fisher face (PCA + Fisher LDA).

The rest of the paper is as follows. In Section II, photon counting linear discriminant analysis and the two types of decision rules are described. Section III demonstrates the experimental and simulation results, and Section IV presents the conclusions. 


\section{Photon Counting Linear Discriminant Analysis}

The Pc-LDA realizes the Fisher criterion using the Poisson distribution which characterizes the semi-classical photodetection model [14]. A random vector $\mathbf{y}$ can be considered a new feature vector corresponding to a normalized image vector $\mathbf{x}$. The dimensions of $\mathbf{x}$ and $\mathbf{y}$ are the same, which is the number of pixels $d$. Each component of $\mathbf{y}$ follows the independent Poisson distribution with the parameter $N_{p} x_{i}$, that is, $y_{i} \sim$ Poisson $\left(N_{p} x_{i}\right) ; x_{i}$ is the normalized intensity at a pixel $i$ such that $\sum_{i=1}^{d} x_{i}=1$, and $N_{p}$ is the total number of average photocounts since $\sum_{i=1}^{d} E\left(y_{i}\right)=\sum_{i=1}^{d} N_{p} x_{i}=N_{p}$.

The between-class covariance measures the separation of classes as

$$
\begin{aligned}
\Sigma_{y y}^{B} & =E_{j}\left[\left(\boldsymbol{\mu}_{y \mid j}-\boldsymbol{\mu}_{y}\right)\left(\boldsymbol{\mu}_{y \mid j}-\boldsymbol{\mu}_{y}\right)^{t}\right] \\
& =N_{p}^{2} \Sigma_{x x}^{B},
\end{aligned}
$$

where the class-conditional mean and the mean vectors are derived as $\boldsymbol{\mu}_{y \mid j}=N_{p} \boldsymbol{\mu}_{x \mid j}$, and $\boldsymbol{\mu}_{y}=N_{p} \boldsymbol{\mu}_{x}$, respectively, and $j$ indicates a class, superscript $t$ denotes matrix transpose. The within-class covariance matrix measures the concentration of members in the same class as

$$
\begin{aligned}
\sum_{y y}^{W} & =E_{j}\left\{E_{y \mid j}\left[\left(\mathbf{y}-\boldsymbol{\mu}_{y \mid j}\right)\left(\mathbf{y}-\boldsymbol{\mu}_{y \mid j}\right)^{t} \mid j\right]\right\} \\
& =N_{p} \operatorname{diag}\left(\boldsymbol{\mu}_{x}\right)+N_{p}^{2} \Sigma_{x x}^{W} .
\end{aligned}
$$

In consequence, the following Fisher criterion can be derived:

$$
\begin{aligned}
W_{P} & =\underset{W \in R^{d \times r}}{\arg \max } \frac{\left|W^{t} \Sigma_{y y}^{B} W\right|}{\left|W^{t} \Sigma_{y y}^{W} W\right|} \\
& =\underset{W \in R^{d \times r}}{\arg \max } \frac{\left|W^{t} \Sigma_{x x}^{B} W\right|}{\left|W^{t}\left[\operatorname{diag}\left(\boldsymbol{\mu}_{x}\right) / N_{p}+\Sigma_{x x}^{W}\right] W\right|},
\end{aligned}
$$

where the column vectors of $W_{P}$ are equivalent with the eigenvectors of $\left[\operatorname{diag}\left(\boldsymbol{\mu}_{x}\right) / N_{P}+\Sigma_{x x}^{W}\right]^{-1} \hat{\Sigma}_{x x}^{B}$ corresponding to the non-zero eigenvalues. It is noted that $\left[\operatorname{diag}\left(\boldsymbol{\mu}_{x}\right) / N_{P}+\Sigma_{x x}^{W}\right]$ is non-singular because of the non-zero components of $\hat{\boldsymbol{\mu}}_{x}$.

The class decision can be made by maximizing a score function as the following:

$$
\hat{j}={ }_{j=1 \ldots . . c}^{\arg \max } \operatorname{score}(j),
$$

where $c$ is the number of classes. The following score functions are derived which are based on Euclidean distance or normalized correlation.

$$
\begin{gathered}
\operatorname{score}_{y}(j)=-\left\|W_{P}^{t}\left(\mathbf{y}_{u}-\boldsymbol{\mu}_{y \mid j}\right)\right\|, \\
\operatorname{score}_{y}(j)=\frac{\left(W_{p}^{t} \mathbf{y}_{u}\right)^{t}\left(W_{p}^{t} \boldsymbol{\mu}_{y \mid j}\right)}{\left\|W_{P}^{t} \mathbf{y}_{u}\right\|\left\|W_{p}^{t} \boldsymbol{\mu}_{y \mid j}\right\|} .
\end{gathered}
$$

The photo-counting vector $\mathbf{y}_{u}$ of an unlabeled object is required for class decision in Eqs. (5) and (6). Alternatively, $\mathbf{y}_{u}$ can be estimated with the intensity image vector $\mathbf{x}_{u}$. Since the minimum mean-squared error (MMSE) estimation is the conditional mean [15], a point estimation of $y_{u i}$ becomes $E\left(y_{u i} \mid x_{u i}\right)=N_{p} x_{u i}$, where $y_{u i}$ and $x_{u i}$ are the $i$-th component of $\mathbf{y}_{u}$ and $\mathbf{x}_{u}$, respectively. In consequence, Eqs. (5) and (6) are equivalent with the score functions in Eqs. (7) and (8), respectively:

$$
\begin{gathered}
\operatorname{score}_{x}(j)=-\left\|W_{P}^{t}\left(\mathbf{x}_{u}-\boldsymbol{\mu}_{x \mid j}\right)\right\|, \\
\operatorname{score}_{x}(j)=\frac{\left(W_{p}^{t} \mathbf{x}_{u}\right)^{t}\left(W_{p}^{t} \boldsymbol{\mu}_{x \mid j}\right)}{\left\|W_{P}^{t} \mathbf{x}_{u}\right\| W_{p}^{t} \boldsymbol{\mu}_{x \mid j} \|} .
\end{gathered}
$$

The mean-squared error is the same with the variance of $y_{u i}$, which is $N_{p} x_{u i}$. The MS error increases as $N_{p}$ increases, however the Pc-LDA converges to the Fisher LDA as $N_{p}$ goes to the infinite as

$$
\lim _{N_{p} \rightarrow \infty} \frac{\left|W^{t} \Sigma_{x x}^{B} W\right|}{\left|W^{t}\left[\operatorname{diag}\left(\boldsymbol{\mu}_{x}\right) / N_{p}+\Sigma_{x x}^{W}\right] W\right|}=\frac{\left|W^{t} \Sigma_{x x}^{B} W\right|}{\left|W^{t} \Sigma_{x x}^{W} W\right|} .
$$

In order to evaluate the performance of the classifiers, two performance measures are employed. One is probability of correct decision $\left(P_{D}\right)$ and the other is probability of false alarm $\left(P_{F A}\right)$ [11]. They are, respectively defined as

$$
\begin{gathered}
P_{D}(j)=\frac{\text { Number of decision for class } j}{\text { Number of test images in class } j}, \\
P_{F A}(j)=\frac{\text { Number of decision for class } j \text {, but are not in class } j}{\text { Number of test images in all classes except for class } j} .
\end{gathered}
$$

In the experiments, the average $P_{D}$ and $P_{F A}$ over call classes are calculated.

\section{Experimental and Simulation Results}


This section describes two types of experiments. The first is for the verification of Pc-LDA with artificially generated random vectors. In the second type of experiments, face recognition tests are performed to evaluate the robustness of Pc-LDA.

\subsection{Pc-LDA verification}

In the first type of experiments, three sets (classes) of threedimensional multivariate Gaussian random variables are generated. The mean vectors of the three classes are set to [5 0 $5]^{t},\left[\begin{array}{lll}-5 & -5 & 0\end{array}\right]^{t}$, and $\left[\begin{array}{lll}5 & -5 & 0\end{array}\right]^{t}$. The covariance matrix of all classes is the $3 \times 3$ identity matrix. One hundred random vectors are generated for each class. Ten vectors per class are trained, and the other 90 vectors per class participate in the decision process. Figure 1(a) represents the Pc-LDA projection results when $N_{p}=$ 10. Figures 1 (b) and 1(c) show the results for $N_{p}=100$ and $N_{p}$ $=1000$, respectively. Figure 1 (c) is almost identical to Figure 1(d), which is the Fisher LDA projection. As $N_{p}$ increases, the decision boundary of Pc-LDA becomes more similar to that of Fisher LDA.

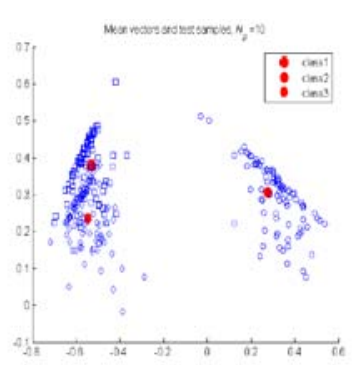

(a)

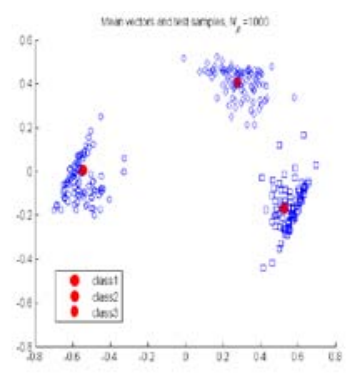

(c)

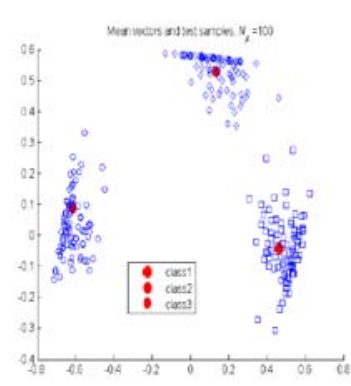

(b)

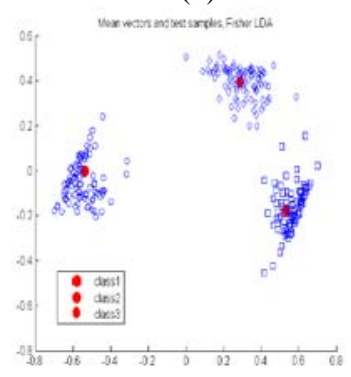

(d)
Figure 1. (a) Pc-LDA when $N_{p}=10$, (b) $N_{p}=100$, (c) $N_{p}=1000$, (d) Fisher LDA, filled marks are the mean vectors of training data.

\subsection{Face recognition}

The "ORL" facial image database [16] is used in the second experiment. This database is composed of 40 classes (persons), each of which comprises 10 facial images captured with different pose, illumination, or expression. The image size is 92 $\times 112$ pixels. The first three images of each class are trained, and the other seven images are provided as unknown inputs. Figure 2 shows the training image samples of classes 1 to 10 .
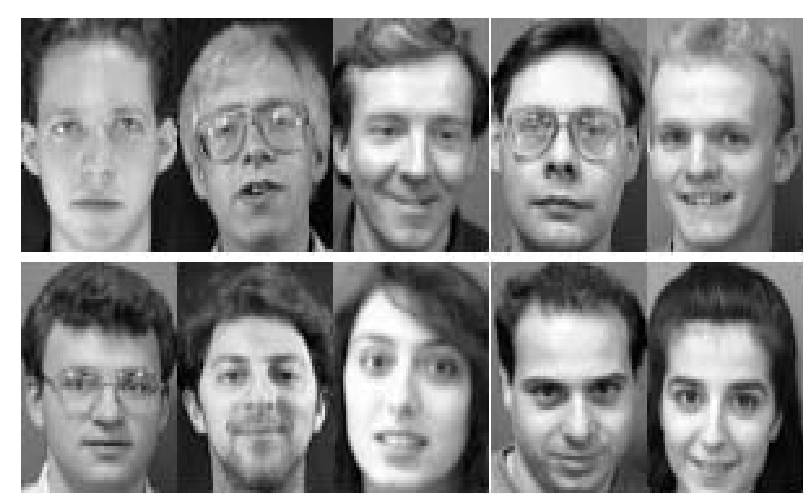

Figure 2. Image samples (class 1-10) in ORL database.

Figures 3(a)-(c) represent discriminant vectors of the PcLDA, Fisher face, and Eigen face, respectively, in the image format. As seen in the figures, Pc-LDA is better at representing the structural diversity, whereas the Eigen face method relies the most on the energy distribution. Thus, Pc-LDA can construct the decision boundary with higher discrimination capability than the other methods.

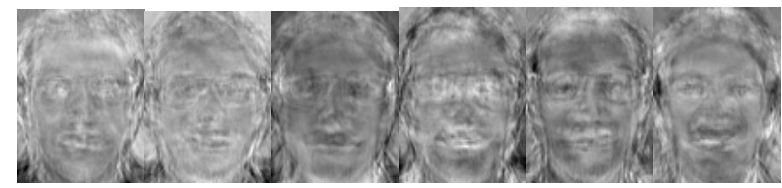

(a)

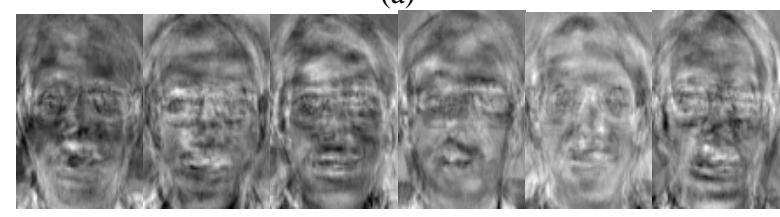

(b)

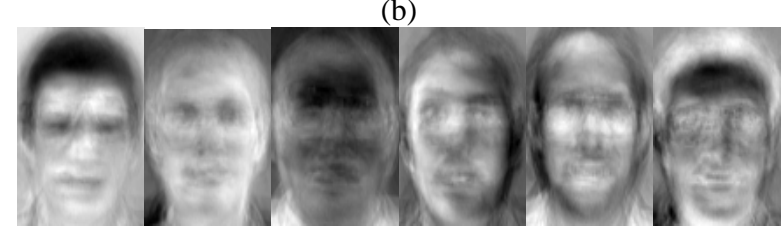

(c)

Figure 3. (a) Pc-LDA face, (b) Fisher face, (c) Eigen face.

The average $P_{D}$ and $P_{F A}$ are obtained in order to compare the recognition performance. Figures 4 and 5 show the recognition results obtained with the Euclidean distance and normalized correlation decision rules, respectively. Cases 1-4 represent the number of trained classes, which are 10, 20,30, and 40. As shown in the figures, Pc-LDA outperforms the other classifiers. The normalized correlation affords better performance than Euclidean metric for Pc-LDA. It can be thought that the normalized correlation is more suitable since it measures the deviation of the direction on the optimal subspace whereas the Euclidian metric compares the absolute distance to the class means regardless of their locations. 


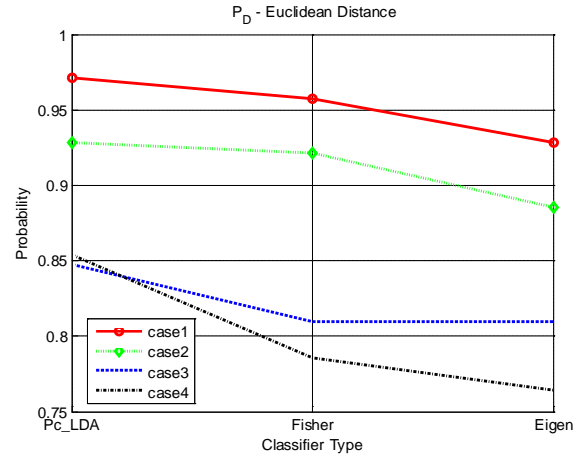

(a)

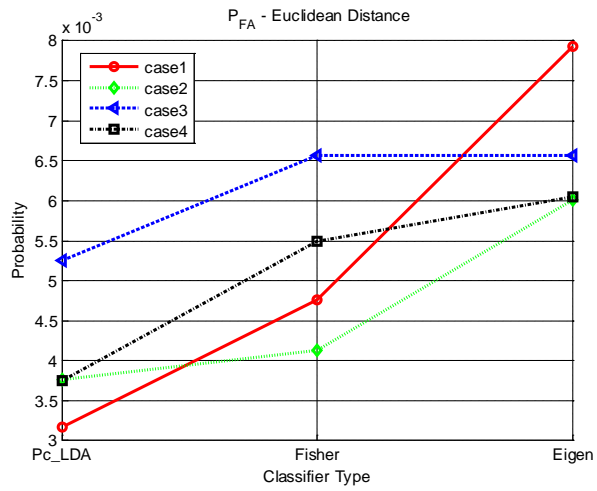

(b)

Figure 4. Classification results with the Euclidean distance decision rule, (a) $P_{D}$, (b) $P_{F A}$.

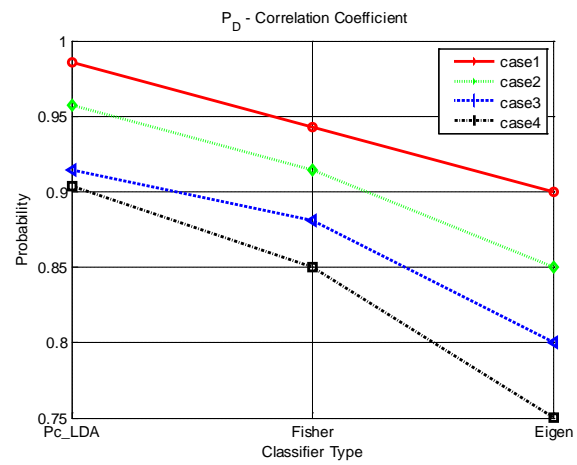

(a)

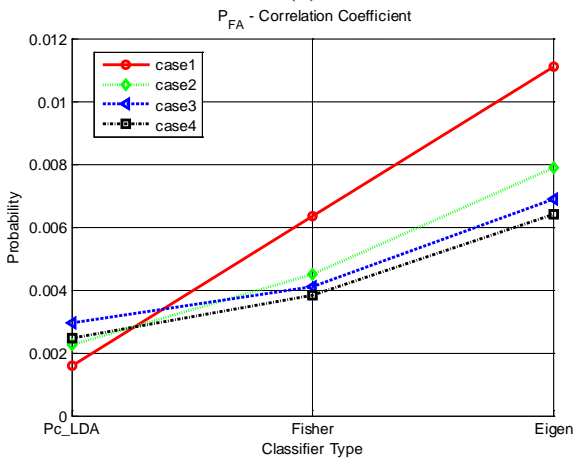

(b)

Figure 5. Classification results with the normalized correlation decision rule, (a) $P_{D}$, (b) $P_{F A}$.
In order to simulate long-distance acquisition, test images are blurred by out-of-focus and motion blurring PSFs as illustrated in figure 6. Types 1 and 2 comprise images rendered out-offocus by applying circular averaging with small and large radius sizes. Types 3 and 4 comprise images that have undergone light and heavy motion blurring, respectively. The radius size of Types 1 and 2 are 4 and 8 pixels, respectively. Types 3 and 4 involve a filter to approximate the linear motion of a camera by $l$ pixels, with an angle of $\Theta$ degrees in a counter-clockwise direction [17]. The parameters are set at 10 pixels and $22.5^{\circ}$, and 20 pixels and $45^{\circ}$, for light and heavy motion blurring, respectively. Figures 7 and 8 show the recognition results for the normalized correlation decision rule for case 2 and case 4, respectively.

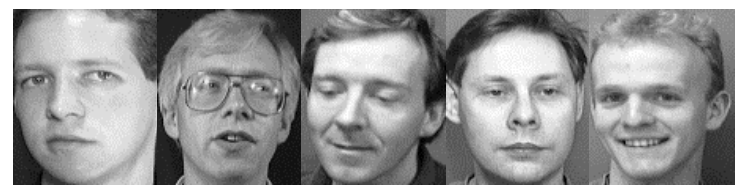

(a)

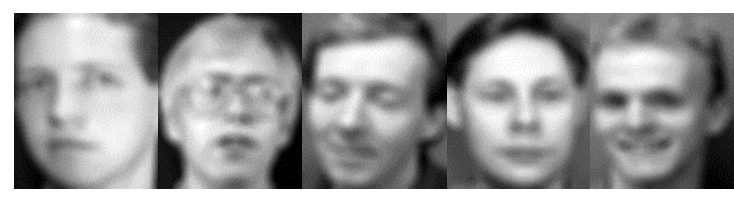

(b)

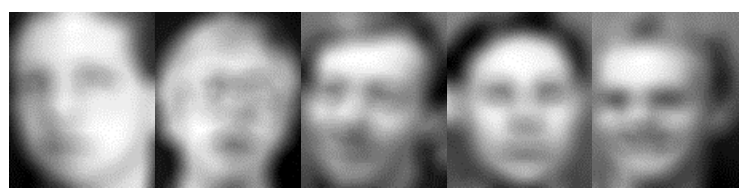

(c)

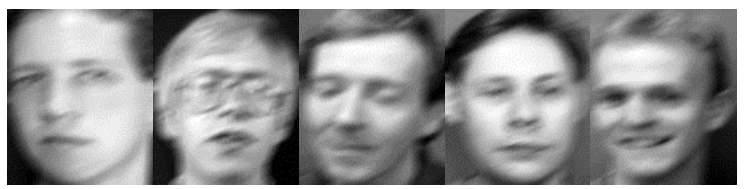

(d)

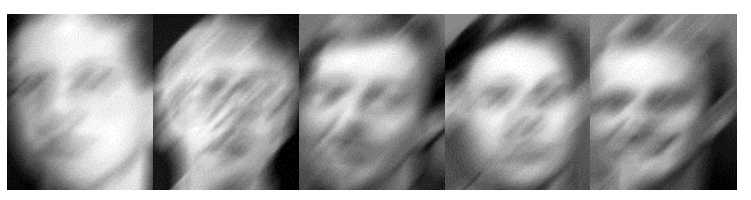

(e)

Figure 6. Test image samples (class 1-5) for (a) No blurring, (b) Type 1, (c) Type 2, (d), Type 3, (e) Type 4.

Figures 7 and 8 show the recognition results with the normalized correlation decision rule. The results with 20 classes are trained in figure 7 and all of 40 classes are trained in figure 8. As illustrated in figures 7 and 8, Pc-LDA is more tolerant to the image distortion than the other methods, 
especially for images rendered heavier blurring in Types 2 and 4.

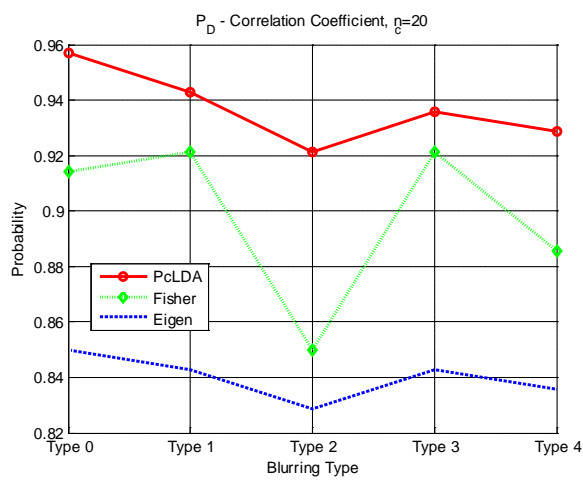

(a)

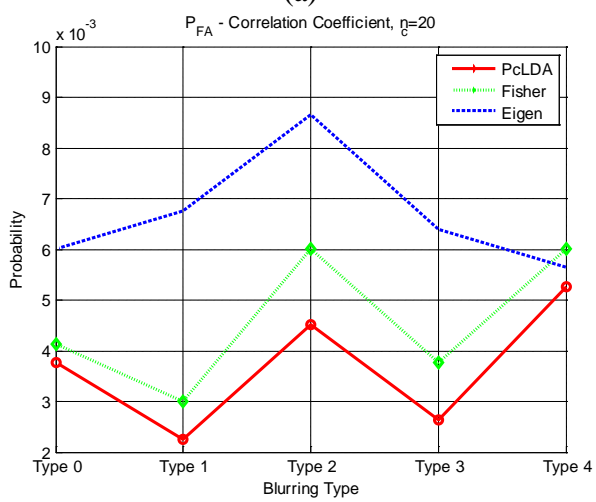

(b)

Figure 7. Classification results for degraded images (Case 2), (a) $P_{D}$, (b) $P_{F A}$.

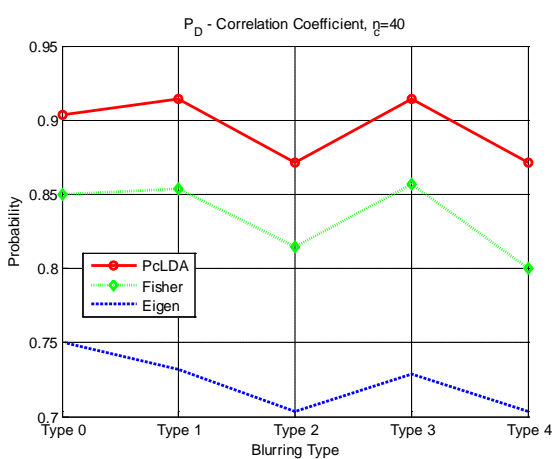

(a)

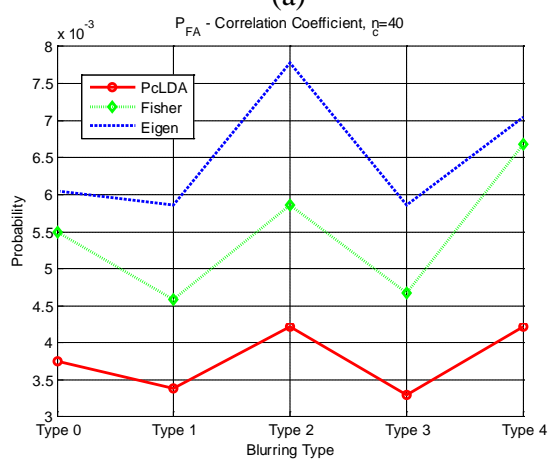

(b)
Figure 8. Classification results for degraded images (Case 4), (a) $P_{D}$, (b) $P_{F A}$.

\section{Conclusion}

This study investigated the effectiveness of a facial recognition system based on Pc-LDA when applied to images captured in harsh environments. Two decision rules, Euclidean distance and normalized cross correlation, were employed. It was found that Pc-LDA overcame the SSS problem when realizing the Fisher criterion, without dimensionality reduction, and afforded robust performance in facial recognition in highly degraded images. The results indicate that Pc-LDA is better than conventional facial recognition techniques.

\section{References}

[1] W. Zhao, R. Chellappa, P. J. Phillips, and A. Rosenfeld, "Face recognition: A literature survey," ACM Computing Surveys, vol. 35, no. 4, pp. 399-458, 2003.

[2] R. Jafri and H. R. Arabnia, "A Survey of Face Recognition Techniques,” Journal of Information Processing Systems, vol. 5, No. 2, pp. 41-68, 2009.

[3] P. N. Belhumer, J. P. Hespanha, D. J. Kriegman, "Eigenfaces vs. Fisherfaces: recognition using class specific linear projection," IEEE Trans. Pattern Analysis and Machine Intelligence, vol. 19, no. 7, pp. 711-720, 1997.

[4] D. L. Swets and J. Weng, "Using discriminant eigenfeatures for image retrieval,” IEEE Trans. Pattern Analysis and Machine Intelligence, vol. 18, pp. 831-836, 1996.

[5] J. H. Friedman, "Regularized discriminant analysis," J. Am. Statistical Assoc., vol. 84, no. 405, pp. 165-175, 1989.

[6] L. J. Park, "A spatial regularization of LDA for face recognition,” International Journal of Fuzzy Logic and Intelligent Systems, vol. 10, no. 2, pp. 95-100, 2010.

[7] S. Raudys and R. P. W. Duin, "Expected classification error of the Fisher linear classifier with pseudo-inverse covariance matrix,” Pattern recognition letters, vol. 19, pp. 385-392, 1998.

[8] B. -H. Moon and K. -B. Sim, "Emotion recognition method based on multimodal sensor fusion algorithm," International Journal of Fuzzy Logic and Intelligent Systems, vol. 8, no. 2, pp. 105-110, 2008.

[9] Y.-H. Cho, "Face recognition by using FP-ICA based on secant method," International Journal of Fuzzy Logic and Intelligent Systems, vol. 5, no. 2, pp. 131-135, 2005.

[10] R. O. Duda, P. E. Hart, and D. G. Stork, Pattern Classification, 2nd ed., Wiley Interscience, New York (2001).

[11] K. Fugunaga, Introduction to Statistical Pattern Recognition, Academic Press (1990).

[12] S. Yeom, B. Javidi, E. Watson, "Three-dimensional distortion-tolerant object recognition using photoncounting integral imaging,” Optics Express, vol. 15, no. 4, 
pp. 1513-1533, 2007.

[13] S. Yeom, "A linear discriminant analysis for low resolution face recognition,” Proc. of 2008 Second International conference on Future Generation Communication and Networking Symposia, pp. 230-233, 2008.

[14] J. W. Goodman, Statistical Optics, Jonh Wiley \& Sons Inc., 1985.
[15] A. Papoulis, Probability, Random Variables, and Stochastic Processes, 3rd ed., McGraw-Hill, Inc., 1991.

[16] ORL Face Database, AT\&T Laboratories Cambridge, 2005.

[17] MATLAB function reference, MathWorks.

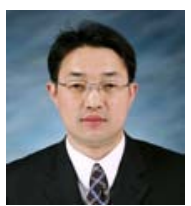

Seokwon (Sekwon) Yeom is currently an assistant professor in Division of Computer and Communication Engineering, Daegu University. He has a Ph.D. in Electrical and Computer Engineering from the University of Connecticut, and an M.S. degree in Electronics Engineering from Korea University and B.S. degrees in Electronics Engineering from Korea University and Physics from Inha University. His research interests include machine learning, signal and image processing, and optical information processing.

E-mail : yeom@daegu.ac.kr 